PREPRINT (IN PRESS, ACCEPTED APRIL 9, 2018). CITE AS:

Burleigh, T. J. \& Rubel, A. N. (in press). Counting polyamorists who count: Prevalence and definitions of an under-researched form of consensual nonmonogamy. Sexualities.

\title{
Counting polyamorists who count: Prevalence and definitions of an under-researched form of consensual nonmonogamy
}

\author{
Alicia N. Rubel \\ Department of Psychology, Brock University, St. Catharines, Canada \\ Correspondence may be sent to Alicia Rubel at ar10hd@brocku.ca. \\ Tyler J. Burleigh \\ Department of Psychology, University of Guelph, Guelph, Canada
}




\begin{abstract}
Despite a growing interest in polyamory, it is unknown how many polyamorists there are in the general population. In acknowledging that the meaning of 'polyamory' is contested (e.g., Klesse, 2014), we estimated the prevalence of polyamory when it was defined as: 1) an identity, 2) relationship beliefs/preferences, 3) relationship status, and 4) relationship agreements. We recruited 972 individuals from Mechanical Turk and used a sample weighting procedure to approximate a representative sample of the United States population. Point prevalence estimates ranged from about $0.6 \%$ to $5 \%$, and lifetime estimates ranged from about $2 \%$ to $23 \%$. Thus, we estimate that there are at least 1.44 million adults in the US who count as polyamorous.
\end{abstract}

Keywords: polyamory, consensual nonmonogamy, prevalence, identity, relationships 
Broadly defined, polyamory is the practice of, belief in, or willingness to engage in multiple simultaneous romantic and/or sexual relationships with the consent of everyone involved. The interest in polyamory is growing, as evidenced by an increasing usage in the English corpora as well as in the scholarly literature (see Figure 1). Indeed, between 1984 and 2015 there have been at least 42 popular nonfiction books published on the topic, seven of which were published in 2014 and 2015 alone (M, 2014a). However, despite this growing interest, it is still unknown how many polyamorists there are in the general population. This is complicated by the multiplicity of definitions of polyamory (Klesse, 2006, 2011, 2014). In the present article, we aim to estimate the prevalence of polyamory according to these various definitions.

[Figure 1 goes here]

To the best of our knowledge, there are only two prevalence estimates of polyamory, and neither has been through a peer-review process of scrutiny. The first estimate, $2.3 \%$, was calculated in 2009 using separate estimates of: 1) the prevalence of polyamory among bisexual individuals, 2) the prevalence of bisexuality among polyamorous individuals, and 3) the prevalence of bisexuality in the general population (M, 2014b). However, this estimate likely has a large margin of error because it is the product of three other estimates, two of which have large individual errors due to their small sample sizes. The second estimate, $7.1 \%$, was obtained in 2015 by the Open Source Psychometrics Project (OSPP, 2015), a website that hosts various psychology tests that are open for anyone to participate in by visiting the website. Although their sample was large $(\mathrm{N}=5043)$ and directly observed, it was also obtained from a population of individuals who take online personality tests, and therefore skewed young (66\% was aged 13$25)$, atheist (47\%), and non-heterosexual (14\%). Given polyamory's cultural and historical 
association with gay and sexual liberation (Easton \& Hardy, 1997; Klesse, 2006, 2011), this estimate is likely to be an overestimate.

Given the overlap between definitions of consensual nonmonogamy and polyamory, the prevalence of consensual nonmonogamy may also be considered as an indicator of polyamory prevalence. Similar to polyamory (when defined as a practice or agreement), consensual nonmonogamy is often defined as a relationship, or relationship agreement, that permits individuals to have more than one sexual and/or romantic relationship at a time, with the knowledge and consent of everyone involved (e.g., Conley et al., 2013). Consensual nonmonogamy is an umbrella term that includes polyamory, but also other relationship practices like swinging and open relationships (Conley et al., 2013; Matsick et al., 2014; Moors et al., 2013). However, as with polyamory prevalence estimation, there have been few attempts to estimate the prevalence of consensual nonmonogamy. Early studies estimated the prevalence of individuals who practice consensual nonmonogamy to be between 4 and $5 \%$ (Conley et al., 2012), and a more recent study which combined Conley et al.'s (2012) dataset with Moors et al.'s (2014) estimated the prevalence to be 5.3\% (Rubin et al., in press). However, in comparison to the general population, the demographics of this sample skewed young (Mage $=27.7$, white (76\%), and female (69\%); and sample weighting was not used to improve sample representativeness. A second limitation is that it is unknown what proportion of the consensual nonmonogamous population is polyamorous, and any estimate of consensual nonmonogamy prevalence is at best a ceiling for the estimate of polyamory prevalence.

Who counts as polyamorous? The task of estimating polyamory prevalence is complicated by the contested nature of the term 'polyamory' (Klesse, 2006). In examining the literature, we encountered many definitions of polyamory (see Table 1), and these definitions 
appeared to cluster into four basic categories: 1) polyamory as beliefs or preferences, 2) polyamory as a relationship status, and 3) polyamory as a relationship agreement. For example, Sheff (2013) adopts a broad stance that acknowledges that polyamory may encompass any one (or more than one) of these aspects, while others have adopted much narrower definitions that only recognize polyamory as ‘something you do' (e.g., Savage, 2012). Further, we observed that in addition to these categories, some definitions have also specified that polyamorous relationships must involve emotional intimacy, romantic love, or be long-term (presumably to distinguish polyamory from other forms of consensual nonmonogamy like swinging; see Klesse, 2006).

[Table 1 goes here]

Depending on which definition is used, a different prevalence estimate would be obtained. For example, a narrow definition of polyamory as someone who is currently in multiple loving relationships would count many fewer individuals than would a broader definition that is based on an individual's openness to having multiple relationships. While we could have proceeded in the present research to use only one of these definitions, it would be difficult to choose. At this point, there is no single conventional definition that is used by members of the polyamorous community, and as such we believe that it is important to conduct research that does not elevate the status of one definition over others. Moreover, by making use of multiple definitions in our research we can gain an understanding of multiple perspectives, practices, and experiences, each of which are interesting in their own right. Thus, we believed that it would be important to examine the prevalence polyamory, based on multiple definitions of polyamory. 
In the present research, we aimed to estimate polyamory prevalence in the general population using the definitions identified above. In addition to this primary goal, we also performed exploratory analyses that examined how polyamory might be defined based on the perspectives and experiences of individuals who identify as polyamorous, in order to inform future research. We believe that estimating the prevalence of polyamory is a worthwhile undertaking for two reasons. First, knowing the prevalence of polyamory can inform our understanding of the diverse ways that humans form and think about romantic and sexual relationships. Second, individuals who practice consensual nonmonogamy are frequently stigmatized and misunderstood (Burleigh et al., submitted; Conley et al., 2012, 2013; GruntMejer \& Campbell, 2016; Hutzler et al., 2015; Moors et al., 2013; Matsick et al., 2014), and face discrimination in many situations, including employment and housing (Emens, 2004;

Fleckenstein, Bergstrand, \& Cox, 2012; Lesher, 2013; Nearing, 2000). Estimating the size of the polyamory population tells us how many individuals in the general population might benefit from public policy and research agendas that address these issues. If this population is relatively large, then the estimate could be used to be used to strengthen the argument that such policies and agendas are valuable by demonstrating that a substantial portion of the population might benefit from them. Although we believe that all minorities are deserving of rights and freedoms regardless of their size, we appreciate the practical significance of this line of argument. Third, this estimate can be used to help service providers to plan for services to polyamorous individuals by letting them know how many individuals might use their services (e.g., sexual health counselling tailored to polyamorists).

\section{Method}

\section{Participants}


A total of 972 participants were recruited from Amazon's Mechanical Turk (MTurk) during the summer of 2013 for a survey that was advertised as being about 'the different styles of relationships that people have'. As eligibility criteria, participants were required to be residents of United States (based on having provided valid taxpayer identification to Amazon), and have had a task approval rate of at least $90 \%$ for previous MTurk tasks (this was used to ensure highquality data; see Peer et al., 2014). We also sought to minimize time-based sample variation by splitting the total data collection into 10 smaller batches and deploying these at different times of day and days of the week (see Casey et al., 2017). ${ }^{1}$ The survey, described in the materials section, took about 10 minutes to complete, and participants were paid \$0.75 USD.

Our decision to use MTurk as a source of data was motivated by several considerations. First, the MTurk population is demographically diverse, and while it is not representative of the general United States population, MTurk samples can be weighted to approximate a representative sample. Previous research has found that Mturk samples are more diverse than other convenience samples like those obtained from student or community populations (Berinsky et al., 2012; Buhrmester et al., 2011; Chandler \& Shapiro, 2016; Paolacci et al., 2010). Although MTurk samples are not demographically representative of the general population, the discrepancy is comparable to samples obtained from professional Internet panels like those obtained by the companies SurveyMonkey and Qualtrics (Heen et al., 2014), and researchers have had success with using poststratification weighting to enhance representativeness (Levay et al., 2016; Simons \& Chabris, 2012). Second, MTurk is cost-effective. According to Mullinix et al. (2015), it can cost more than $\$ 15,000$ to conduct even a brief survey with a population-based

${ }^{1}$ Specifically, we posted 10 batches with 100 slots each at the following days and times (Eastern Standard Time): Sunday at $1 \mathrm{pm}$, Monday at $10 \mathrm{am}$, Tuesday at $11 \mathrm{pm}$, Wednesday at 7:30pm, Thursday at $8 \mathrm{pm}$, Friday at $11 \mathrm{pm}$, Monday at $3 \mathrm{pm}$, and Tuesday at $4 \mathrm{pm}$. 
sample, while a comparable sample can be recruited from MTurk for only $\$ 500$. Finally, MTurk affords anonymity to participants (or at least a sense of anonymity, as participants are technically pseudonymous), and is therefore appropriate for collecting information from individuals who represent stigmatized and concealable identities (Smith et al., 2015) — as is the case with polyamory.

\section{Materials}

The survey asked participants demographic questions (age, sex, ethnicity, household income, education, sexual orientation, and feminist identity) and questions about their relationships. The relationship questions included: 1) relationship status, 2) consensual nonmonogamy identity (e.g., polyamorous), 3) the nature of their relationship agreement(s), and 4) their beliefs about relationships. In order to facilitate both point and lifetime prevalence estimation, participants were asked the relationship questions with respect to both past and present circumstances. Finally, participants were also asked about their knowledge of the term 'polyamory'. Survey branch logic was used to dynamically present relevant questions to participants. For example, only participants who indicated that they were in a relationship were then asked to indicate the characteristics of that relationship. Thus, not all participants answered all questions. See Appendix A for the survey questions and branch logic. To assess the prevalence of polyamory according to the four definitions identified earlier, we examined responses as follows:

1. Identity: To assess polyamory as an identity, we examined responses to a question that asked participants whether or not they identified as: polyamorous/polyamorist, swinger, or non-monogamist (participants were allowed to select multiple options). 
2. Relationship beliefs/preferences: To assess polyamory as relationship beliefs/preferences, we examined responses to the question: 'I currently believe that it is [In the past I believed that it was] for me personally [a partner of mine/people in general] to have more than one romantically intimate relationship at a time, with the knowledge and consent of everyone involved' (bracketed portions indicate separate questions). Response options were: possible, acceptable, desirable, and none of the above; and participants could select multiple options. We examined responses that indicated the belief that it was: 1) possible, 2) possible and acceptable, and 3) possible and acceptable and desirable.

3. Relationship agreement: To assess polyamory as a relationship agreement, we examined responses to the following questions: 'I am currently in a relationship [In the past, I have been in a relationship] where my partner and I explicitly agree[d] that it [is / was] to have more than one romantically intimate relationship at a time [to have more than one sexual relationship at a time / to experience romantic love for more than one person at the same time], with the knowledge and consent of everyone involved' (bracketed portions indicate separate questions). Response options were: possible, acceptable, desirable, and none of the above; and participants could select multiple options. We examined responses that indicated an agreement where it was: 1) possible, 2) possible and acceptable, and 3) possible and acceptable and desirable. Further, we examined responses to questions that asked participants to describe the relationship(s) in which they had this agreement: sexual, romantic, loving, long-term, intimate, or marriage; and participants could select multiple options. 
4. Relationship status: To assess polyamory as a relationship status, we examined responses to the following questions: 'Are you currently in [In the past, have you ever been in] multiple sexual and/or romantic relationships, with the knowledge and consent of everyone involved?' (Yes/No), and 'Do you [Did you] consider any [more than one] of your multiple simultaneous relationships to be sexual, romantic, loving, long-term intimate and/or a marriage?' Response options were: sexual, romantic, loving, long-term, intimate, and marriage; and participants could select multiple options.

\section{Sample Weighting}

Our analyses were performed using SPSS 22 (IBM Corp, 2013). We used the SPSS rake extension to weight our sample to the 2012 U.S. Census, using age, ethnicity, sex, income, and education as weighting variables. SPSS rake implements a form of poststratification weighting called random iterative method (or iterative proportional fitting; as described in Grover \& Vriens, 2006), that assigns weights to individual cases in order to enhance the representativeness of the sample. Higher weights are given to individuals in the sample who are underrepresented relative to the general population, and lower weights are given to individuals who are overrepresented. For example, if the population distribution of sex was known to be $50 \%$ female / 50\% male, and the sample was 30\% female / 70\% male, then each female in the sample would be given a weight of 1.67 (50/30), and each male would be given a weight of $0.71(50 / 70)$. When multiple weighting variables are used, the method adjusts the sample to the population on all variables simultaneously. By adjusting the sample to match the general population, more valid inferences can be made about characteristics of the general population (Levay et al., 2016; Simons \& Chabris, 2012).

\section{Results}




\section{Demographics}

We first compared the demographics of our sample to those of the 2012 U.S. Census, in order to determine to what extent our sample differed from the general population. When all demographic variables and brackets within variables were considered, the average discrepancy was 5.5 percentage points (age $=6.6$, ethnicity $=5.0$, sex $=4.3$, income $=2.3$, and education $=$ 12.4). The largest individual bracket discrepancies (15-20 percentage points) were observed with age and education, reflecting an overrepresentation of the 20-24 and 25-29 age brackets, and the college and bachelor degree education brackets. In addition, the White (not Hispanic) ethnicity and $\$ 100,000+$ income brackets observed discrepancies between 10-15 percentage points. The remaining discrepancies were less than 10 percentage points each. See Table 2 for a summary. ${ }^{2}$ Given these findings, it seemed reasonable to proceed with the weighted sample analysis.

[Table 2 goes here]

\section{Prevalence of Polyamory}

First, we examined the prevalence of polyamory when defined as an identity. We found that $0.59 \%$ of participants in the weighted sample currently identified as polyamorous or polyamorist, and $1.79 \%$ identified as polyamorous or polyamorist at some point in their life. By comparison, $2.35 \%$ currently identified as swingers, $2.50 \%$ as nonmonogamist, and $5.09 \%$ as any one of the nonmonogamous options; lifetime prevalence was $4.76 \%$ for swinger, $8.81 \%$ for nonmonogamist, and $12.85 \%$ for any one of the nonmonogamous options. ${ }^{3}$

${ }^{1}$ It is also worth noting that $11 \%$ of our sample identified as non-heterosexual $(4.8 \%=$ [gay, lesbian, or homosexual], $8.5 \%=$ [bisexual, queer, pan or polysexual], $1.7 \%=$ questioning, $1.0 \%=$ asexual, and $<1.0 \%=$ demisexual), and $27.3 \%$ identified as feminist (a philosophy associated with polyamory; Haritaworn, Lin, \& Klesse, 2006).

${ }^{3}$ The unweighted estimates were as follows: $1.64 \%$ currently identified as polyamorous/polyamorist; $1.23 \%$ as swingers, $2.57 \%$ as nonmonogamist, and $4.11 \%$ as any one of the nonmonogamous options. For lifetime prevalence, $3.70 \%$ identified as polyamorous/polyamorist; $2.36 \%$ as swingers, $5.75 \%$ as nonmonogamist, and $8.83 \%$ as any one of the nonmonogamous options. 
Second, we examined the prevalence of polyamory when defined as relationship beliefs/preferences. A broad definition might be indicated by the belief that it is both possible and acceptable to have consensual romantic relationships with others. Along these lines, we found that $4.31 \%$ of participants believed that it was possible and acceptable for themselves, and $3.91 \%$ believed that it was possible and acceptable for their partner(s). Lifetime prevalence (beliefs held now or in the past) was $7.92 \%$ for the self and $7.53 \%$ for their partner(s). A narrower definition, which incorporates the notion of preference, would be indicated by the belief that it possible, acceptable, and also desirable to have consensual romantic relationships with others. We found that $2.46 \%$ of participants held these beliefs for themselves, and $1.46 \%$ held these beliefs for their partner(s). Lifetime prevalence was 5.64\% for the self and 3.60\% for their partner(s). For a summary that includes unweighted estimates, see Table $3{ }^{4}$

[Table 3 goes here]

Third, we examined the prevalence of polyamory when defined as a relationship agreement. Among the participants who indicated that they were currently in a relationship, $9.68 \%$ (5.57\% of the total weighted sample; $5.03 \%$ unweighted) answered that they had a consensually nonmonogamous agreement with a partner. Lifetime prevalence (currently or in the past) was $23.00 \%$. Given that some definitions of polyamory specify that love is essential, participants were asked to indicate which adjective(s) they would use to describe the relationship(s) in which they had the consensually nonmonogamous agreement(s). The polyrelevant adjectives were: sexual, romantic, loving, long-term, intimate (marriage was included for reference). That is to say, polyamory might be defined as a relationship that permits

\footnotetext{
${ }^{4}$ Notably, only $39.22 \%$ of the participants stated that it was acceptable for people in general to engage in consensual nonmonogamy, confirming that consensually non-monogamous relationships are frequently devalued.
} 
nonmonogamy which can be described by any one of these adjectives. We found that each of these descriptors was used by about $5 \%$ of the total weighted sample ('sexual' was the highest at $5.33 \%$, and 'loving' was the lowest at $4.84 \%$ ). For lifetime prevalence, 'sexual' was used by $15.04 \%$ of the total weighted sample, 'romantic' and 'intimate' by about $10 \%$, and 'loving' and 'long-term' by about $8 \%$. For a summary that includes unweighted estimates and the marriage descriptor, see Tables $4 \mathrm{a}$ and $4 \mathrm{~b}$.

\section{[Tables $4 \mathrm{a}$ and $4 \mathrm{~b}$ go here]}

A second line of questions that we asked participants to assess polyamorous relationship agreements, was whether they were in a relationship in which they and their partner had explicitly agreed that it was possible, acceptable, and/or desirable to engage in several consensually nonmonogamous behaviours. Recall that polyamory is sometimes defined as loving multiple persons. The behaviours that we asked about were whether they: 1) have more than one consensual romantic relationship at a time, 2) have more than one consensual sexual relationship at a time, and/or 3) consensually experience romantic love for more than one person at the same time. A broad definition of polyamory would be where partners agree that at least one of these three consensually nonmonogamous behaviours is possible and acceptable. Along these lines, we found that about $1.5 \%$ (range: $1.5 \%$ to $1.61 \%$ ) of participants currently had an agreement where at least one of the behaviours was possible and acceptable. For lifetime prevalence, about $8 \%$ had agreed that multiple consensual sexual or romantic relationships were possible and acceptable; whereas only $2.8 \%$ had agreed with their partner that it was possible and acceptable to experience romantic love for more than one person at a time. A narrower definition of polyamory would be the agreement that it is possible, acceptable, and also desirable, to engage in one of the three consensually nonmonogamous behaviours. Here we observed similar point 
estimates: about $1.35 \%$ (range: $1.2 \%$ to $1.44 \%$ ) currently had an agreement where at least one of the behaviours was possible, acceptable, and desirable. For lifetime prevalence, $3.5 \%$ had at some point agreed with a partner that multiple consensual sexual or romantic relationships were possible, acceptable, and desirable; whereas only $1.84 \%$ had at some point agreed with a partner that it was possible, acceptable, and desirable to experience romantic love for more than one person at a time. For a summary that includes unweighted estimates, see Table $4 \mathrm{~b}$.

Finally, we examined the prevalence of polyamory when defined as a relationship status; $4.12 \%$ (2.26\% unweighted) answered that they were currently in multiple consensual sexual and/or romantic relationships. Lifetime prevalence was $18.44 \%$ (16.53\% unweighted). Again, because some definitions specify that polyamorous relationships are loving or long-term in nature, participants were asked to asked to indicate if any [more than one] of their multiple consensually nonmonogamous relationships could be described as sexual, romantic, loving, long-term, or intimate. When asked to describe any of their consensually nonmonogamous relationships, each of the poly-relevant descriptors was used by about $4 \%$ of the total sample; the results were also about $4 \%$ each when participants were asked to describe more than one of their consensually nonmonogamous relationships. In terms of lifetime prevalence, when participants were asked to describe any of their multiple consensually nonmonogamous relationships in the past or present, 'sexual' was used by $18.19 \%$ of the total weighted sample, 'loving' by $12.44 \%$, followed by 'romantic,' 'long-term,' and 'intimate' which were each used by about $8 \%$. When participants were asked to describe more than one of their multiple consensually nonmonogamous relationships in the past or present, 'sexual' was used by $16.91 \%$ of the total weighted sample, 'loving' by $10.61 \%$, 'intimate' and 'romantic' by about $7.5 \%$, and 'long-term' 
by $5.72 \%$. For a summary that includes unweighted estimates and the marriage descriptor, see Table 5.

[Table 5 goes here]

\section{Participants' Understanding of What 'Polyamory' Means}

Importantly, our estimate of the prevalence of polyamorous-identified individuals must be qualified by participants' understanding of what 'polyamory' means, because it is likely that more individuals who meet other definitions of polyamory would identify as polyamorous if they understood what it meant. To assess participants' understanding of polyamory, we coded the responses to the open-ended question that asked participants to define polyamory. Responses were coded as having a basic understanding of polyamory if they mentioned multiple partners (romantic or sexual) or mentioned being in love with multiple individuals, and if they also did not imply that it necessarily involved marriage. For example, one representative response was: "Polyamory is being in love with two or more people at the same time, and being in simultaneous relationships with two or more people at the same time." We also coded responses as having a comprehensive understanding of polyamory if they also mentioned consent in their response. For example, one respondent said polyamory was "A non-monogamous relationship or desire to have one such relationship including multiple romantic partners at the same time, with all partners being aware of the situation."

We chose to code accuracy of the definitions using the above requirements based on our observations of how polyamory has been defined in past research and popular literature (see Table1). Having multiple partners or loves is clearly central to the common understanding of polyamory. In addition, as can be seen by the table, most works emphasize that polyamory refers to consensual nonmonogamy rather than surreptitious behaviour. This focus on consent and 
honesty is typically seen as a key principle of polyamory (e.g., Emens, 2004). Although there are some debates regarding how polyamory should be defined, whether or not it involves nonconsensual nonmonagamy (i.e., cheating) is not one of them (Emens, 2004; Klesse, 2006; 2014)

Although some of the participants who gave a correct basic definition of polyamory likely knew that this arrangement requires consent but excluded this information for the sake of brevity, including a more restrictive comprehensive category gives us a more conservative estimation of the number of people who are familiar with the definition of polyamory. Interrater agreement acceptable (Cohen, 1960) on both coding variables (basic understanding $\kappa=.996$; comprehensive understanding $\kappa=.991)$. We found that $38.6 \%$ of participants $(51.7 \%$ unweighted) reported a basic understanding of polyamory, and 14.7\% (13.6\% unweighted) reported a comprehensive understanding of polyamory.

\section{What does Polyamory Mean to Polyamorists?}

Although our sample of self-identified polyamorists was small $(\mathrm{N}=16)$, we believed that it would be worth examining what polyamory meant to these individuals. Thus, we performed two exploratory analyses on the unweighted sample to provide preliminary data relevant to this question.

First, we examined responses to a question that asked polyamorous-identified participants what polyamory meant to them ('to me, polyamory is'). The response options were: 1) 'innate (i.e., some people are born polyamorous)', 2) 'a system of beliefs', 3) 'a practice or lifestyle (i.e., something that you do)', 4) 'something you feel', and 5) 'none of the above'; and participants could select multiple options. We found that $94 \%$ of polyamorists stated that polyamory was a practice or lifestyle, $63 \%$ stated that it was a system of beliefs, $50 \%$ stated that it was something one feels, $44 \%$ stated that it was innate, and $6 \%$ stated that it was none of these options. 
Next, we examined to what extent the non-identity based aspects of polyamory could predict polyamorous self-identification. We performed a hierarchical logistic regression in which the dependent variable was identification as 'polyamorous' or 'polyamorist' vs. neither (coded as 0 or 1). We began by entering the broadest criteria that could be used to define polyamory, and then at each successive step we added progressively more restrictive criteria. To reduce potential multicollinearity and to limit the number of predictors in the model, some of the definitional factors were combined into ordinal variables and others were excluded. Specifically, we entered: 1) beliefs about what is possible and acceptable for people in general (general beliefs), 2) beliefs about what is acceptable for oneself and one's partner (personal beliefs), 3) the presence or absence of a consensually nonmonogamous agreement (multiple relationships), 4) the status of being in multiple consensually nonmonogamous relationships (multiple relationships), 5) whether participants loved one of their partners (one love), 6) whether participants had an agreement with their partner where it was possible, acceptable, and/or desirable to love more than one person at a time (multiple love agreement), and 7) whether participants loved more than one of their partners (multiple love). Thus, this regression allowed us to determine at what steps using an increasingly restrictive definition of polyamory better predicted identification as polyamorous/a polyamorist.

The results of the regression were as follows. At Step 1, the belief that polyamory is acceptable for people in general significantly predicted polyamorous identification $(B=.97, p=$ .008). At Step 2, personal beliefs about the acceptability of polyamory significantly predicted polyamorous identification $(B=1.36, p<.001)$, while general beliefs were no longer significant $(B=.09, p=.841)$. The addition of variables in Steps 3-7 did not improve the predictive ability of the model as none of the variables reached significance ( $p s>.05)$. Correlations between the 
predictors in our model are presented in Table 6, and a summary of model coefficients is presented in Table 7.

[Tables 6 and 7 go here]

\section{Discussion}

\section{Prevalence}

In the present study, we sought to estimate polyamory prevalence when polyamory was defined in several different ways. Specifically, when polyamory was defined as: 1) an identity, 2) relationship beliefs/preferences, 3) relationship status, and 4) relationship agreements. We found that the point and lifetime prevalence estimates varied based on how broadly or narrowly polyamory was defined. The point estimates ranged from about $0.6 \%$ to about $5 \%$, while the lifetime estimates ranged from about $2 \%$ to $23 \%$. The lowest point estimates were observed when polyamory was defined as an identity, while the highest point estimates were observed when polyamory was defined as relationships with a consensual nonmonogamy agreement which could be described as either sexual, romantic, intimate, loving, or long-term (each of which obtained similar estimates). Similarly, the lowest lifetime estimates were observed when polyamory was defined as an identity, while the highest lifetime estimate was observed when polyamory was defined as relationships with a consensual nonmonogamy agreement which participants described as sexual. Finally, intermediate prevalence estimates were observed when polyamory was defined as a relationship that permitted (or permitted and encouraged) different kinds of consensually nonmonogamous behaviours (sexual relationships, romantic relationships, or consensually loving multiple people), and when polyamory was defined as relationship beliefs. Thus, the question of how polyamory is defined is an important consideration for the task of prevalence estimation. Given both these findings and the contested nature of the term 
'polyamory' (e.g., Klesse, 2006), we suggest that researchers should avoid elevating the status of any one definition of polyamory, and instead describe the prevalence of polyamory using multiple definitions or ranges.

Importantly, our data on the prevalence of polyamory as an identity must be qualified by participants' understanding of 'polyamory' as a concept. We found that only about $39 \%$ of individuals had a basic understanding of polyamory (as involving multiple partners or loving multiple individuals), and only about $15 \%$ reported a comprehensive definition (as also involving consent). Insofar as the polyamorous-identified population tracks understanding of polyamory as a concept, this suggests that this population is likely to grow over time with increasing awareness.

As awareness grows, and social attitudes change, researchers should again estimate the prevalence of polyamory. Our estimates were derived from a convenience sample collected on the MTurk platform - a decision that was motivated by practical limitations like cost. We nonetheless believe the sample to have provided more robust estimates than those obtained in previous efforts. We made every effort to control sampling bias (as with our efforts to minimize time-based variation and our use of sample weighting), however despite these efforts our sample could still have had unknown biases. For these reasons, future estimates should be derived from a probability sample.

\section{Defining Polyamory}

Beyond the task of identifying polyamory prevalence, our data also contributes to an ongoing debate about the nature of polyamory - that is, whether polyamory is 'something you do' or 'something you are' (Barker, 2005; Savage, 2012), with the latter case typically being seen as comparable to 'sexual orientation'. Such a distinction is believed to have several 
practical implications. Defining polyamory as 'something you are' may help to achieve social acceptance, legal protections, custody rights, marriage equality, and other improvements in the treatment of polyamorists in society (Aviram \& Leachman, 2015; Emens, 2004; Klesse, 2014; 2016; Tweedy, 2011). For example, Tweedy (2011) argued that members of a group are more likely to succeed with anti-discrimination claims if they can make the case that their situation is analogous to that of an oppressed racial group, which involves emphasizing how the group membership or identity is a core aspect of who they are (e.g., an immutable trait).

However, there are practical disadvantages to reducing polyamory to an immutable trait or absorbing it into the umbrella of 'sexual orientation' (Aviram \& Leachman, 2015; Emens, 2004; Klesse, 2014; Klesse, 2016). Limiting the definition of polyamory in this way could fragment the social movements that polyamorists engage in, threaten the status of gender and sexual minorities, and undermine the more radical and transformative ideologies that polyamory is associated with. For example, Klesse $(2014 ; 2016)$ argues that if polyamorists advocate for their rights using a restricted definition of polyamory, then any protections gained this way would apply only to a small minority of individuals, and those who fall outside of this definition may be pressured to conform in order to protect the image of polyamory. Alliances that exist between polyamorists, other consensual nonmonogamists, and individuals who have nontraditional families in other ways (e.g., single-headed families) would be lost. Essentially, polyamorists would be more likely abandon their broader critique of relationship norms in favour of advocating for rights and protections for a select few.

Regardless of its practical implications, our results suggest that, for many polyamorists, polyamory can be seen not only as something that someone "does" (e.g., form multiple relationships), but as something a person "is", similar to a sexual identity (a potentially innate 
preference) or a religious identity (a deeply held set of beliefs). Specifically, we found that of individuals who identified as polyamorous or a polyamorist $(\mathrm{N}=16), 63 \%$ stated the opinion that polyamory was a set of beliefs, and $44 \%$ stated that polyamory was innate. Indeed, in our regression, relationship status did not predict polyamorous identity over and above beliefs. We would again argue for the advantages of a multiplicity of definitions, and for honouring the ways that polyamorous individuals define their own experiences of polyamory.

\section{Conclusion}

Based on the most conservative polyamory prevalence estimate of $0.6 \%$ (those who explicitly identified as polyamorous or a polyamorist), and given that the size of the U.S. adult (18+) population was estimated to be 240 million (U.S. Census Bureau, 2012), it follows that there are at least 1.44 million adults in the U.S. population who could be classified as polyamorous. As a point of comparison, Gates (2011) estimated that there were about 4 million individuals (1.7\%) who identified as bisexual, 4 million (1.8\%) who identified as lesbian or gay, and 700 thousand $(0.3 \%)$ who identified as transgender; Bogaert (2004) estimated that $1.04 \%$ of the population meets the criteria for asexuality. Therefore, by our most conservative estimate, there are at least twice as many polyamorists as transgender individuals, and by our most inclusive estimates, there are many more polyamorists than gay or lesbian individuals. Thus, polyamorous individuals represent a substantial portion of the population.

\section{Acknowledgements}

This research was supported by funding from the Ontario Public Interest Research Group, OPIRG-Guelph (opirgguelph.org) and OPIRG-Brock (opirgbrock.com) chapters. We would also like to thank Danielle Molnar for generously giving her time to instruct us on the use of rake weights. 


\section{Statement of Research Ethics}

This research was approved by the Research Ethics Board at Brock University (File \#12252). 


\section{Reference List}

Anderlini-D’Onofrio, S. (2004: 164-165). Polyamory, in J. Eadie (Ed.) Sexuality: The Essential Glossary. London: Arnold.

Anderson, S., Wandersee, S., Arcenas, A., \& Baumgartner, L. (2013). Craigslist samples of convenience: recruiting hard-to-reach populations. Retrieved September 6, 2016 from University of California Santa Barbara: http://fiesta.bren.ucsb.edu/ sanderson/CraigslistSurvey.pdf.

Aviram, H., \& Leachman, G. M. (2015). The future of polyamorous marriage: Lessons from the marriage equality struggle. Harvard Women's Law Journal, 38, 269.

Barker, M. (2005). This is my partner, and this is my... partner's partner: Constructing a polyamorous identity in a monogamous world. Journal of Constructivist Psychology, 18(1), 75-88. doi: 10.1080/10720530590523107

Berinsky, A. J., Huber, G. A., \& Lenz, G. S. (2012). Evaluating online labor markets for experimental research: Amazon.com's Mechanical Turk. Political Analysis, 20(3), 351368. doi: 10.1093/pan/mpr057

Bogaert, A. F. (2004). Asexuality: Prevalence and associated factors in a national probability sample. Journal of Sex Research, 41(3), 279-287. doi: 10.1080/00224490409552235

Buhrmester, M., Kwang, T., \& Gosling, S. D. (2011). Amazon's Mechanical Turk a new source of inexpensive, yet high-quality, data? Perspectives on Psychological Science, 6(1), 3-5. doi: $10.1177 / 1745691610393980$

Burleigh, T. J., Rubel, A. N., \& Meegan, D. V. (2017). Wanting 'the whole loaf': Zero-sum thinking about love is associated with prejudice against consensual non- 
monogamists. Psychology \& Sexuality, 8(1-2), 24-40. doi:

$10.1080 / 19419899.2016 .1269020$

Casey, L. S., Chandler, J., Levine, A. S., Proctor, A., \& Strolovitch, D. Z. (2017). Intertemporal differences among MTurk workers: Time-based sample variations and implications for online data collection. SAGE Open, 7(2). doi: 10.1177/2158244017712774

Chandler, J., \& Shapiro, D. (2016). Conducting clinical research using crowdsourced convenience samples. Clinical Psychology, 12(1), 53-81. doi: 10.1146/annurev-clinpsy021815-093623

Cohen, J. (1960). A coefficient of agreement for nominal scales. Educational and Psychological Measurement, 20(1), 37-46. doi: 10.1177/001316446002000104

Conley, T. D., Moors, A. C., Matsick, J. L., \& Ziegler, A. (2012). The fewer the merrier?: Assessing stigma surrounding consensually non-monogamous romantic relationships. Analyses of Social Issues and Public Policy, 13(1) 1-29. doi: 10.1111/j.15302415.2012.01286.x

Conley, T. D., Ziegler, A., Moors, A. C., Matsick, J. L., \& Valentine, B. (2013). A critical examination of popular assumptions about the benefits and outcomes of monogamous relationships. Personality and Social Psychology Review, 17(2), 124-41. doi: $10.1177 / 1088868312467087$

CPAA. (2013). About [the CPAA]. Retrieved February 2, 2013, from Canadian Polyamory Advocacy Association, http://polyadvocacy.ca/about

Easton, D., \& Hardy, J. W. (2009). Ethical slut: A practical guide to polyamory, open relationships \& other adventures (2nd ed.). Berkeley, CA: Celestial Arts. 
Emens, E. (2004). Monogamy's law: Compulsory monogamy and polyamorous existence. University of Chicago Public Law, Working Paper. Retrieved September 17, 2016 from https://papers.ssrn.com/sol3/papers.cfm?abstract _id=506242

Fleckenstein, J., Bergstrand, C. R., \& Cox, D. W. I. (2012). What do polys want?: An overview of the 2012 Loving More survey. Retrieved from https://www.lovemore.com/polyamoryarticles/2012-lovingmore-polyamory-survey

Gates, G. J. (2011, April 1). How many people are lesbian, gay, bisexual and transgender? Retrieved September 17, 2016, from The Williams Institute, http://williamsinstitute.law.ucla.edu/research/census-lgbt-demographics-studies/howmany-people-are-lesbian-gay-bisexual-and-transgender/

Grover, R., \& Vriens, M. (2006). The handbook of marketing research: uses, misuses, and future advances. London: SAGE Publications.

Grunt-Mejer, K., \& Campbell, C. (2016). Around consensual nonmonogamies: Assessing attitudes toward nonexclusive relationships. The Journal of Sex Research, 53(1), 45-53. doi: 10.1080/00224499.2015.1010193

Heen, M. S., Lieberman, J. D., \& Miethe, T. D. (2014). A comparison of different online sampling approaches for generating national samples. Retrieved September 17, 2016 from Center for Crime and Justice Policy, https://www.unlv.edu/sites/default/files/page_files/27/ComparisonDifferentOnlineSampli ng.pdf

Haupert, M., Gesselman, A., Moors, A., Fisher, H., \& Garcia, J. (2016). Prevalence of experiences with consensual non-monogamous relationships: Findings from two 
nationally representative samples of single Americans. Journal of Sex \& Marital

Therapy, 1-17. doi: 10.1080/0092623x.2016.1178675

Haritaworn, J., Lin, C. J., \& Klesse, C. (2006). Poly/logue: A critical introduction to polyamory. Sexualities, 9(5), 515-529. doi: 10.1177/1363460706069963

Hutzler, K. T., Giuliano, T. A., Herselman, J. R., \& Johnson, S. M. (2016). Three's a crowd: public awareness and (mis)perceptions of polyamory. Psychology \& Sexuality, 7(2), 6987. doi: 10.1080/19419899.2015.1004102

IBM Corp (2013). IBM SPSS Statistics for Windows [software], Version 22.0. Armonk, NY: IBM Corp.

Klesse, C. (2006). Polyamory and its 'others': Contesting the terms of non-monogamy. Sexualities, 9(5), 565-583. doi: 10.1177/1363460706069986

Klesse, C. (2011). Notions of love in polyamory_Elements in a discourse on multiple loving. Laboratorium. 2, 4-25.

Klesse, C. (2014). Polyamory: Intimate practice, identity or sexual orientation? Sexualities, 17(12), 81-99. doi: 10.1177/1363460713511096

Klesse, C. (2016). Marriage, Law and Polyamory. Rebutting mononormativity with sexual orientation discourse? Oñati Socio-legal Series, 6(6), 1348-1376.

Lesher, E. C. (2013). Protecting poly: Applying the fourteenth amendment to the nonmonogamous. Tulane Journal of Law \& Sexualty, 22, 127-145.

Levay, K. E., Freese, J., \& Druckman, J. N. (2016). The demographic and political composition of Mechanical Turk samples. SAGE Open, 6(1). doi: 10.1177/2158244016636433 
M, A. (2014a, August 31). All 42 nonfiction books on modern polyamory. Retrieved September

5, 2016, from Polyamory in the News, http://polyinthemedia.blogspot.ca/2014/08/all-39nonfiction-books-on-modern.html

M, A. (2014b, May 27). 'Are Bisexuals the Glue that Holds Poly Together?' And some numbers for how many polys there are. Retrieved September 3, 2016, from Polyamory in the News, http://polyinthemedia.blogspot.ca/2009/04/are-bisexuals-glue-that-holds-poly.html

Matthesen, E. (1997). Alt.Polyamory frequently asked questions (FAQ). Retrieved February 02, 2013, from alt.polyamory [usenet], http://www.faqs.org/faqs/polyamory/faq/

Michel, J.-B., Shen, Y. K., Aiden, A. P., Veres, A., Gray, M. K., Pickett, J. P. Hoiberg, D. Clancy, D. Norvig, P., Orwant, J., Pinker, S., Nowak, M. A., Aiden, E. L. \& The Google Books Team (2011). Quantitative analysis of culture using millions of digitized books. Science, 331(6014), 176-182. doi: 10.1126/science.1199644

Moors, A. C., Conley, T. D., Edelstein, R. S., \& Chopik, W. J. (2014). Attached to monogamy? Avoidance predicts willingness to engage (but not actual engagement) in consensual nonmonogamy. Journal of Social and Personal Relationships, 32(2), 222-240. doi:

$10.1177 / 0265407514529065$

Moors, A. C., Matsick, J. L., Ziegler, A., Rubin, J. D., \& Conley, T. D. (2013). Stigma toward individuals engaged in consensual nonmonogamy: Robust and worthy of additional research. Analyses of Social Issues and Public Policy, 13(1), 52-69. doi: 10.1111/asap. 12020

Mullinix, K. J., Leeper, T. J., Druckman, J. N., \& Freese, J. (2015). The generalizability of survey experiments. Journal of Experimental Political Science, 2(2), 109-138. doi: $10.1017 / x p s .2015 .19$ 
Nearing, R. (2000). Polyamory demography - the 'Loving More Magazine' study. Retrieved from http://www.kinseyinstitute.org/resources/Nearing.html

OSPP (2015) The demographics of polyamory/monogamy from a general population survey. Retrieved September 3, 2016, from Open Source Psychometrics Project, http://personality-testing.info/research/demographics-of-polyamory

Papadakis, M. (2015, February 17). 'Polyamorous' woman loses fight with Catholic church. Retrieved September 16, 2016, from The Sydney Morning Herald, http://www.smh.com.au/national/polyamorous-woman-loses-fight-with-catholic-church20150217-13gtv9.html

Paolacci, G., Chandler, J., \& Ipeirotis, P. (2010). Running experiments on Amazon Mechanical Turk. Judgment and Decision Making, 5(5), 411-419.

Peer, E., Vosgerau, J., \& Acquisti, A. (2014). Reputation as a sufficient condition for data quality on Amazon Mechanical Turk. Behavior Research Methods, 46(4), 1023-1031. doi: $10.3758 / \mathrm{s} 13428-013-0434-\mathrm{y}$

Polyamory.org.uk. (2013). Introduction to Polyamory. Retrieved February 02, 2013, from Polyamory.org.uk, http://www.polyamory.org.uk/polyamory_intro.html

Rubin, J. D., Moors, A. C., Matsick, J. L., Ziegler, A., \& Conley, T. D. (in press). On the margins: Considering diversity among consensually non-monogamous relationships. Journal für Psychologie.

Savage, D. (2012, November 21). Savage love. Retrieved September 6, 2016, from http://www.thestranger.com/seattle/SavageLove?oid=15339984

Sexualities (2003). Special Issue on Polyamory - Call for Contributors. Sexualities, 6(1), 126. 
Sheff, E. (2013). The polyamorists next door: Inside multiple-partner relationships and families. Lanham, Maryland: Rowman \& Littlefield.

Simons, D. J., \& Chabris, C. F. (2012). Common (mis) beliefs about memory: A replication and comparison of telephone and Mechanical Turk survey methods. PLoS ONE, 7(12), e51876.

Smith, N. A., Sabat, I. E., Martinez, L. R., Weaver, K., \& Xu, S. (2015). A convenient solution: Using MTurk to sample from hard-to-reach populations. Industrial and Organizational Psychology, 8(2), 220-228. doi: 10.1017/iop.2015.29

Tweedy, A. E. (2011). Polyamory as a sexual orientation. University of Cincinnati Law Review, $79,1461$.

U.S. Census Bureau. (2009). National characteristics: Vintage 2012. Retrieved September 16, 2016 from https://www.census.gov/popest/data/national/asrh/2012

van Anders, S. M. (2015). Beyond sexual orientation: Integrating gender/sex and diverse sexualities via sexual configurations theory. Archives of Sexual Behavior, 44(5), 11771213. doi: 10.1007/s10508-015-0490-8

Veaux, F., Hardy, J., \& Gill, T. (2014). More than two: A practical guide to ethical polyamory. Portland, OR: Thorntree Press, LLC.

Weitzman, G. (2006). Therapy with clients who are bisexual and polyamorous. Journal of Bisexuality, 6(1-2), 137-164. doi: 10.1300/j159v06n01_08 